\title{
A Novel $\mathbf{T}^{2}$-SVM for Partially Supervised Classification
}

\author{
Lorenzo Bruzzone and Mattia Marconcini \\ Dept. of Information and Communication Technology, University of Trento, \\ Via Sommarive, 14, I-38050, Povo, Trento, Italy \\ Phone: +39-0461-882056, Fax: +39-0461-882093 \\ lorenzo.bruzzoneding.unitn.it
}

\begin{abstract}
This paper addresses partially supervised classification problems, i.e. problems in which different data sets referring to the same scenario (phenomenon) should be classified but a training information is available only for some of them. In particular, we propose a novel approach to the partially supervised classification which is based on a Bi-transductive Support Vector Machines ( $\mathrm{T}^{2}$-SVM). Inspired by recently proposed Transductive SVM (TSVM) and Progressive Transductive SVM (PTSVM) algorithms, the $\mathrm{T}^{2}$-SVM algorithm extracts information from unlabeled samples exploiting the transductive inference, thus obtaining high classification accuracies. After defining the formulation of the proposed $\mathrm{T}^{2}$-SVM technique, we also present a novel accuracy assessment strategy for the validation of the classification performances. The experimental results carried out on a real remote sensing partially supervised problem confirmed the reliability and the effectiveness of both the $\mathrm{T}^{2}$-SVM and the corresponding validation procedure.
\end{abstract}

\section{Introduction}

In the pattern recognition literature, classification problems are usually addressed according to supervised or unsupervised approaches, depending on both the availability of prior information and the specific investigated application. If a reliable training set is available, supervised methods are preferred, otherwise it is mandatory to adopt unsupervised clustering techniques. However, there are also hybrid problems which lie between these two opposite families. In this context, a novel issue concerns partially supervised classification problems [1], [2]. The term partially supervised is used to indicate situations in which many data sets referring to the same scenario (phenomenon) are available, but a reliable training set is not available for all of them. This is a common situation in several real applications (e.g., video surveillance, remote sensing, speech recognition), where gathering reliable prior information is often too expensive both in terms of economic costs and time. The aim of partially supervised techniques is to obtain an acceptable classification accuracy for all the considered data sets, included those for which a specific training set is not available. Therefore, the investigated problem becomes strongly ill-posed and ill-conditioned, because no labeled samples are available for some data sets.

In this paper, we addressed the aforementioned problem in the framework of Support Vector Machines (SVMs). SVMs proved to be very effective in addressing standard supervised classification problems. Recently, the Transductive SVM (TSVM) [3] 
and Progressive Transductive SVM (PTSVM) [4] algorithms, which exploit the transductive inference (i.e., extracting information from the spectral distribution of unlabeled samples), proved capable to improve the classification accuracies with respect to standard inductive SVM when only few training samples are available. In order to take advantage of the transductive inference in addressing the partially supervised problem, inspired by both TSVMs and PTSVMs, we propose a bi-transductive SVM (called $\mathrm{T}^{2}$-SVM) that, starting from an inductive learning inference, is able to update the decision function according to a double transductive inference based on unlabeled samples. In particular, a three-step procedure is defined by considering the different types of data that are taken into account (only training samples, only transductive samples or both of them).

Given the complexity of the investigated problem, the accuracy assessment of the classification algorithm plays a very important role, considering that: i) the problem is strongly ill-posed; ii) standard statistical methods for validation cannot be employed because no labeled samples are available for defining a test set. For this reason, in this work we also present a novel empirical validation strategy, which assumes that there exist an intrinsic structure underlying a classification process that rules the partially supervised problem.

The paper is organized into six sections. Section 2 describes the simplifying assumptions and the notation adopted, whereas Section 3 addresses the proposed $\mathrm{T}^{2}$-SVM technique. Section 4 introduces the derived validation approach. Section 5 reports the experimental results, and, finally, Section 6 draws the conclusions.

\section{Problem Formulation}

In the following, without loss of generality, we consider as an example of partially supervised problem the classification of two different data sets, $\mathbf{R}_{1}$ and $\mathbf{R}_{2}$ (e.g. two remote sensing images, two audio signals), referring to the same scene observed at different times ( $t_{1}$ and $t_{2}$, respectively). We assume that prior information is available only for $\mathbf{R}_{1}$. It is worth nothing that the extension to the case of more data sets is straightforward.

Let $\mathbf{X}_{1}=\left\{\mathbf{x}_{1}^{1}, \mathbf{x}_{2}^{1}, \ldots, \mathbf{x}_{M}^{1}\right\}$ and $\mathbf{X}_{2}=\left\{\mathbf{x}_{1}^{2}, \mathbf{x}_{2}^{2}, \ldots, \mathbf{x}_{N}^{2}\right\}$ denote two proper subsets of $\mathbf{R}_{1}$ and $\mathbf{R}_{2}$ composed of $M$ labeled and $N$ unlabeled patterns, respectively. We refer to $\mathbf{X}_{1}$ as "training set" and to $\mathbf{X}_{2}$ as "transductive set", whereas we call $\mathbf{x}_{1}^{2}, \ldots, \mathbf{x}_{N}^{2}$ "transductive patterns". Let $\mathbf{x}_{i}^{1}$ and $\mathbf{x}_{i}^{2}$ be the $1 \times d$ feature vectors associated with the $i$-th sample of $\mathbf{X}_{1}$ and $\mathbf{X}_{2}$ (where $d$ represents the dimensionality of the input space) and let $A=\left|\mathbf{X}_{1}\right|$ be the cardinality of $\mathbf{X}_{1}$. We assume that in our system the set of classes that characterize the considered data sets is fixed; therefore only the spatial and spectral distributions of such classes are supposed to vary over time. This assumption is reasonable in several real applications [1], [2]. We name $\Omega=\left\{\omega_{1}, \omega_{2}, \ldots, \omega_{L}\right\}$ the set of $L$ classes that characterize the two data sets at both $t_{1}$ and $t_{2} ; y_{i}^{1}, y_{i}^{2} \in \Omega$ are the classification labels of the $i$-th pattern of $\mathbf{X}_{1}$ and $\mathbf{X}_{2}$, respectively. In the formulation of the proposed technique, we make the following assumptions: 
- a set of training labels $\mathbf{Y}_{1}=\left\{y_{1}^{1}, y_{2}^{1}, \ldots, y_{M}^{1}\right\}$ for $\mathbf{X}_{1}$ is available;

- a set of training labels $\mathbf{Y}_{2}=\left\{y_{1}^{2}, y_{2}^{2}, \ldots, y_{N}^{2}\right\}$ for $\mathbf{X}_{2}$ is not available;

- the statistical distribution of the classes in $\mathbf{R}_{1}$ and $\mathbf{R}_{2}$ is consistent.

Under the aforementioned hypothesis, our goal is to perform an accurate and robust classification of $\mathbf{R}_{2}$ by exploiting $\mathbf{X}_{1}, \mathbf{Y}_{1}$ and $\mathbf{X}_{2}$.

\section{The Proposed T²-SVM Technique}

The proposed technique is divided into three main phases, which refer to the different types of samples exploited in the learning process: i) only training patterns (inductive inference); ii) training and transductive patterns (bi-transductive inference); iii) only transductive patterns (algorithm convergence). For the sake of simplicity, we formulate the $\mathrm{T}^{2}$-SVM technique addressing a two-class problem.

\subsection{Phase 1: Inductive Inference}

The initial step of the entire process $(t=0)$ corresponds to the first phase of the $\mathrm{T}^{2}$-SVM technique. In order to determine the starting position of the separating hyperplane, at the beginning a standard inductive SVM is applied only to training samples of the first data set (acquired at time $t_{1}$ ). As we assume to deal with linearly non-separable data, the inductive SVM solves the following minimization problem [5]:

$$
\left\{\begin{array}{c}
\min _{\left(\mathbf{w}^{(0)}, b^{(0)}, \xi^{(0)}\right)}\left\{\frac{1}{2}\left\|\mathbf{w}^{(0)}\right\|^{2}+C^{(0)} \sum_{i=1}^{A^{(0)}} \xi_{i}^{(0)}\right\} \\
y_{i}^{1} \cdot\left(\mathbf{w}^{(0)} \cdot \mathbf{x}_{i}^{1}+b^{(0)}\right) \geq 1-\xi_{i}^{(0)}, \quad \forall i=1, \ldots, A^{(0)}, \mathbf{x}_{i}^{1} \in \mathbf{X}_{1}^{(0)}
\end{array}\right.
$$

where $\xi_{i}^{(0)}$ are positive slack variables, $C^{(0)}$ is the regularization parameter, $\mathbf{w}^{(0)}$ represents the vector normal to the separating hyperplane and $b^{(0)}$ is the bias of the separating hyperplane [5].

The value of $C^{(0)}$ can be chosen a priori depending on the standard methods commonly used in literature [5]. All the transductive patterns $\mathbf{x}_{1}^{2}, \ldots, \mathbf{x}_{N}^{2}$ are classified according to the resulting decision function $f^{(0)}(\mathbf{x})=\mathbf{w}^{(0)} \cdot \mathbf{x}+b^{(0)}$.

\subsection{Phase 2: Bi-transductive Inference}

The second iterative phase represents the core of the proposed algorithm. At the generic iteration $t$, for all the transductive patterns the corresponding value of the decision function determined at the iteration $t-1$ is computed. The $P$ unlabeled transductive samples lying into the margin band which are closest both to the lower or the upper margin bound are given the label " -1 " and " +1 ", respectively ( $P$ is a free parameter defined a priori by the user). It may happen that the number of unlabeled patterns in one side of the margin is lower than $P$. In such situations the labeling is done anyway. As a consequence, let $D \leq P$ and $U \leq P$ denote the number of transductive patterns labeled at the current iteration which belong to the lower and the upper side of the margin, respectively. 
The main purpose of the $\mathrm{T}^{2}-\mathrm{SVM}$ technique is to define and solve a bound minimization problem involving only the patterns that describe the classification problem at $t_{2}$. Consequently, at each iteration a subset of the training samples of the first data set is deleted. In particular, from the original training set $\mathbf{X}_{1}^{(0)}$, the $D$ samples with label " -1 " and the $U$ samples with label " +1 " furthest from the separating hyperplane are erased (they are the training patterns that less affect the position of the separating hyperplane). Figure 1 and Figure 2 show an example on a simulated data set. If the training set is not completely erased yet and the margin band is empty, the number of patterns to delete becomes $E$. This parameter is defined a priori by the user and is not critical. It is worth nothing that in this way the position of the separating hyperplane changes at each iteration, therefore a dynamical adjusting is mandatory. It may happen, in fact, that the classification results at the current iteration do not match the labels assigned earlier: if the new label is different from the previous one, the transductive pattern is reset as unlabeled. As it will be underlined in the following, our aim is to gradually increase the regularization parameter for the transductive patterns that algorithm for a simulated data set. Training patterns $\mathbf{x}_{i}^{1} \in \mathbf{X}_{1}$ are shown as white and black circles; transductive patterns $\mathbf{x}_{i}^{2} \in \mathbf{X}_{2}$ are shown as grey squares. The separating hyperplane is shown as a solid line, while the dashed lines define the margin. The dashed circles highlight the $P(P=3)$ transductive patterns selected from both the upper and the lower side of the margin labeled at the first iteration. The dashed squares surround the training patterns to erase. have been given a label, according to a time-dependent criterion. We say that all the samples of $\mathbf{X}_{2}^{(0)}$ which have been assigned until iteration $t-1$ always to the same label, belong to set $\mathbf{J}^{(t)}$. This set is partitioned into a finite number of subsets $G$, where $G$ is a free parameter called Growth Rate and represents the maximum number of iterations for which the user

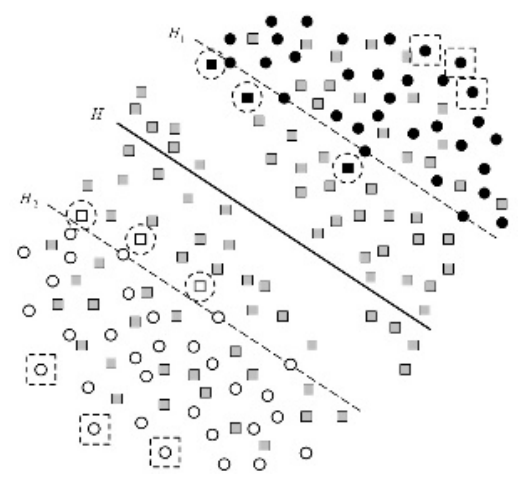

Fig. 1. Margin and separating hyperplane resulted after the first phase $(t=0)$ of the $\mathrm{T}^{2}-\mathrm{SVM}$ algorithm for a simulated data set. Training patterns $\mathbf{x}_{i}^{1} \in \mathbf{X}_{1}$ are shown as white and black circles; transductive patterns $\mathbf{x}_{i}^{2} \in \mathbf{X}_{2}$ are shown as grey squares. The separating hyperplane is shown as a solid line, while the dashed lines define the margin. The dashed circles highlight the $P(P=3)$ transductive patterns selected from both the upper and the lower side of the margin labeled at the first iteration. The dashed squares surround the training patterns to erase. 
allows the regularization parameter to increase. Each subset $\mathbf{J}_{l}^{(t)}$ contains all the samples that belong to the subset with index $l-1$ at iteration $t-1$ and are consistent with the results of the current separating hyperplane. The cost function to minimize and the corresponding bounds become the following:

$$
\left\{\begin{array}{c}
\min _{\left(\mathbf{w}^{(t)}, b^{(t)}, \xi^{(t)}, \xi^{(t)}\right)}\left\{\frac{1}{2}\left\|\mathbf{w}^{(t)}\right\|^{2}+C^{(t)} \sum_{i=1}^{A^{(t)}} \xi_{i}^{(t)}+\sum_{l=1}^{G} C_{l}^{*} \sum_{j=1}^{B_{l}^{(t)}} \xi_{l j}^{*(t)}\right\} \\
y_{i}^{1} \cdot\left(\mathbf{w}^{(t)} \cdot \mathbf{x}_{i}^{1}+b^{(t)}\right) \geq 1-\xi_{i}^{(t)}, \forall i=1, \ldots, A^{(t)} \\
\left(y_{l j}^{2}\right)^{(t)} \cdot\left(\mathbf{w}^{(t)} \cdot \mathbf{x}_{l j}^{2}+b^{(t)}\right) \geq 1-\xi_{l j}^{*(t)}, \forall l=1, \ldots, G, \forall j=1, \ldots, B_{l}^{(t)}
\end{array}\right.
$$

where $\mathbf{x}_{i}^{1} \in \mathbf{X}_{1}^{(t)}, \mathbf{x}_{l j}^{2} \in \mathbf{J}_{l}^{(t)} \subseteq \mathbf{X}_{2}^{(0)}$ and

$$
\left\{\begin{array}{l}
\tilde{\xi}^{*(t)}=\left\{\xi_{11}^{*(t)}, \ldots, \xi_{1, B_{1}}^{*}{ }^{(t)}, \ldots, \xi_{G, 1}^{*}{ }^{(t)}, \ldots, \xi_{G, B_{G}}^{*}{ }^{(t)}\right\} \\
B_{l}^{(t)}=\left|\mathbf{J}_{l}^{(t)}\right|, \quad \forall l=1, \ldots, G \\
\left(y_{l j}^{2}\right)^{(t)}=f^{(t-1)}\left(\mathbf{x}_{l j}^{2}\right), \quad \forall l=1, \ldots, G, \forall j=1, \ldots, B_{l}^{(t)}
\end{array}\right.
$$

At the generic iteration $t$, the algorithm associates the patterns of $\mathbf{J}^{(t)}$ with a regularization parameter $C_{l}^{*}$ depending on the $l$-th subset $\mathbf{J}_{l}^{(t)}$. The regularization parameter $C^{(t)}$ corresponding to the original training samples that describe the classification problem at $t_{1}$, changes at each iteration. A proper choice for $C^{(t)}$ and $C_{l}^{*}$ has an important role for an effective behavior of the algorithm. In order to decrease the influence of the patterns of $\mathbf{X}_{1}^{(0)}$ in determining the position of the separating hyperplane, at each iteration, on the one end, we let their cost-factor $C^{(t)}$ drop gradually down. On the other hand, in order to better control possible mislabelings, we let the regularization parameter associated with the transductive patterns gradually increase. Training

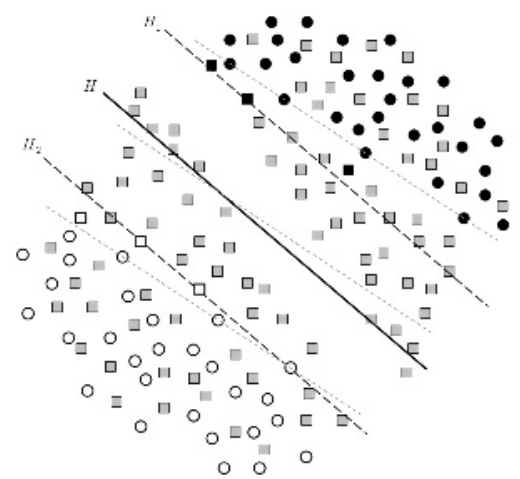

Fig. 2. Margin and separating hyperplane resulting at the end of the first iteration $(t=1)$ of the $\mathrm{T}^{2}$-SVM algorithm. The dashed grey lines represent both the separating hyperplane and the margin at the beginning of the learning process. 
and transductive data might exhibit very different distribution functions. As a consequence, we reasonably expect that a transductive pattern starts assuming a significant weight during the learning process only after it has the same label for some iterations. Accordingly, we decided to increase the cost-factor for the transductive patterns in a quadratic way, depending on the number of cycles they last inside $\mathbf{J}$. Likewise, we let the cost factor for the training samples, $C^{(t)}$, decrease in a quadratic way too. The described procedure requires that three parameters have to be chosen a priori: $C \equiv C^{(0)}$, $C^{*} \equiv C_{1}^{*}$ and $G$ (with the constraint $C>C^{*}$ ). At step $G$, we make $C^{(t)}$ and $C_{l}^{*}$ assume the values $C^{*}$ and $C_{G}^{*}$, respectively. In order to better control the classification error, we set $C_{G}^{*}=C / 2$. The second phase ends at iteration $K$, when $\mathbf{X}_{1}^{(0)}$ is empty.

\subsection{Phase 3: Algorithm Convergence}

The second phase ends when all the original training patterns associated to $\mathbf{R}_{1}$ have been erased. Consequently, in the last phase, only the transductive samples are considered. We can rewrite the initial minimization problem as follows:

$$
\left\{\begin{array}{c}
\min _{\left(\mathbf{w}^{(t)}, b^{(t)}, \xi^{*(t)}\right)}\left\{\frac{1}{2}\left\|\mathbf{w}^{(t)}\right\|^{2}+\sum_{l=1}^{G} C_{l}^{*} \sum_{j=1}^{B_{l}^{(t)}} \xi_{l j}^{*(t)}\right\} \\
\left(y_{l j}^{2}\right)^{(t)} \cdot\left(\mathbf{w}^{(t)} \cdot \mathbf{x}_{l j}^{2}+b^{(t)}\right) \geq 1-\xi_{l j}^{*(t)}, \forall l=1, \ldots, G, \forall j=1, \ldots, B_{l}^{(t)}
\end{array}\right.
$$

When both the remaining unlabeled samples of $\mathbf{X}_{2}^{(0)}$ lying into the margin band at the current iteration and the number of mislabeled patterns with respect to the previous iteration are lower than or equal to $\lceil a \cdot N\rceil$, we assume that convergence is reached and denote the corresponding iteration $t=\operatorname{con} v$. The coefficient $a$ is fixed a priori and tunes the sensitivity of the learning process (a reasonable empirical choice has proven to be $a=0.02$ ). At the iteration $t=c o n v+1$ the final minimization problem becomes the following:

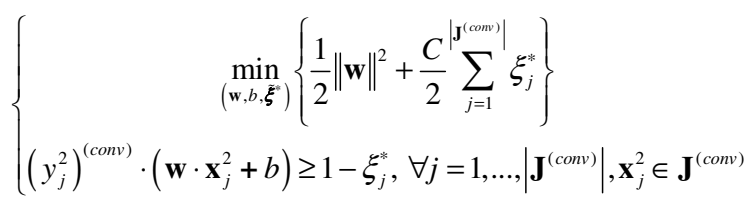

The aforementioned problem is solved by employing a standard inductive SVM because the algorithm associates to all the patterns the same cost-factor $C / 2$. Now, all the patterns of $\mathbf{R}_{2}$ can be labeled according to the current separating hyperplane (see Figure 3). Note that $\mathbf{X}_{2}$ and $\mathbf{R}_{2}$ might coincide. The bigger is $\mathbf{X}_{2}$, the higher is the number of transductive patterns taken into account in positioning the separating hyperplane and the more accurate becomes the classification of $\mathbf{R}_{2}$. However, an effective trade-off is necessary, because the computational load increases together with the size of $\mathbf{X}_{2}$.

The above-described algorithm is defined only for two-class problems. When a multiclass problem has to be investigated, the choice of adopting a One-Against-All 
strategy [5] is mandatory. At each iteration, in fact, all the transductive patterns should be labeled. Hence, we cannot employ a One-Against-One strategy: it is not possible to consider only samples that are supposed to belong to two specific information classes without labeling all the others.

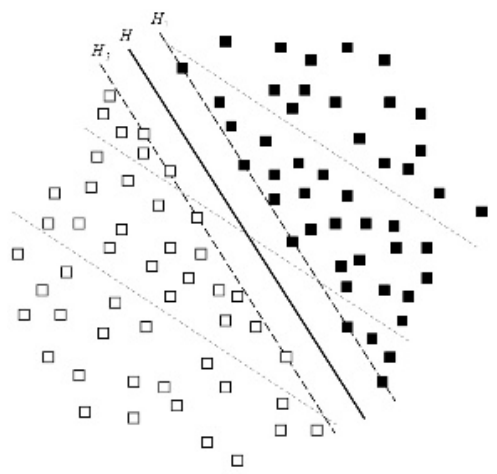

Fig. 3. Final margin and separating hyperplane resulting after the third phase of the $\mathrm{T}^{2}-\mathrm{SVM}$ algorithm. The dashed grey lines represent both the separating hyperplane and the margin at the beginning of the learning process.

\section{The Proposed Accuracy Assessment Strategy}

As we pointed out before, since no prior information for the second data set $\mathbf{R}_{2}$ is available, a technique for validating the classification results of the $\mathrm{T}^{2}$-SVM technique is necessary. We investigated such challenging issue assuming that there exist an intrinsic structure which underlies the classification process and relates the solutions to the partially supervised problem that are consistent for $\mathbf{R}_{1}$ and $\mathbf{R}_{2}$. Let us suppose to obtain an acceptable solution for $\mathbf{R}_{2}$ by exploiting a $\mathrm{T}^{2}-\mathrm{SVM}$. Our idea is that by applying again the $\mathrm{T}^{2}$-SVM algorithm in the reverse sense (using the classification labels in place of the missing prior knowledge for $\mathbf{R}_{2}$ and keeping the same transductive parameters), in the most cases it is possible to obtain a reasonable classification accuracy over $\mathbf{R}_{1}$. At the beginning, we train an inductive SVM using the labeled patterns $\mathbf{x}_{i}^{1} \in \mathbf{X}_{1}^{(t)}$; the resulting solution is supposed to be acceptable for $\mathbf{R}_{1}$ : we say that the system is in the state $\bar{A}$. Afterwards, we apply the bi-transductive algorithm as described in Section III. For both the kernel-function parameters and the regularization parameter $C$ we keep the same values used in the learning phase of the inductive SVM. If the four parameters $C^{*}, G, P$ and $E$ are set properly, the solution is expected to be consistent for $\mathbf{R}_{2}$ and the system moves to the state $\bar{B}$; otherwise, if the solution is not-consistent, the system moves to the state $\bar{D}$. Let $y_{1}^{2}, \ldots, y_{M}^{2}$ denote the classification labels corresponding to the transductive samples $\mathbf{x}_{i}^{2} \in \mathbf{X}_{2}^{(t)}$. Successively, we apply the $\mathrm{T}^{2}$-SVM algorithm again using $\mathbf{X}_{2}$ with the labels $y_{1}^{2}, \ldots, y_{M}^{2}$ as 
training set and $\mathbf{X}_{1}$ as transductive set (it is worth nothing that the labels $y_{1}^{1}, \ldots, y_{N}^{1}$ are not taken into account for the learning purpose). All the transductive parameters remain the same. Let $C A \in[0 \div 1]$ denote the classification accuracy parameter selected by the user (e.g., the Kappa coefficient, the Overall Accuracy), whereas let $C A_{t h}$ represent a prefixed threshold for it. We can compute the value for $C A$ associated to the results obtained by the $\mathrm{T}^{2}-\mathrm{SVM}$ addressing the reverse bi-transductive process because the labels $y_{1}^{1}, \ldots, y_{N}^{1}$ are known. When $C A<C A_{t h}$, the classification accuracy for $\mathbf{R}_{1}$ is considered non-acceptable and the system moves to the state $\bar{C}$, otherwise the solution is consistent and the system moves back to the state $\bar{A}$. When the system, starting from the state $\bar{A}$, is able to return into the state $\bar{A}$, we can empirically assume that the classification accuracy over $\mathbf{R}_{2}$ is satisfactory and, as a consequence, the solution is acceptable. This aspect is very crucial because in such situations we are able to assess that $\mathbf{R}_{2}$ is classified with a proper accuracy for our classification task even if no a priori information is available. Let us denote $P(\bar{Y} \mid \bar{X})$ be the probability that the system joins the generic state $\bar{Y}$ starting from the generic state $\bar{X}$ by applying the $\mathrm{T}^{2}-\mathrm{SVM}$ algorithm. The proposed validation technique is effective under the two following conditions:

- $\quad P(\bar{A} \mid \bar{D})=0$ and $P(\bar{C} \mid \bar{D})=1$ : if the solution obtained by the first $\mathrm{T}^{2}$-SVM is not acceptable, it must be never possible to obtain an acceptable solution for $\mathbf{R}_{1}$ by applying the bi-transductive algorithm in the reverse sense;

- $\quad P(\bar{A} \mid \bar{B})>0$ : starting from an acceptable solution for $\mathbf{R}_{2}$ it must be possible, by applying the $\mathrm{T}^{2}$-SVM algorithm in the reverse sense, to obtain an acceptable solution for $\mathbf{R}_{1}$. The transductive parameters are not optimized for the reverse bitransductive process, therefore we always assume that $P(\bar{C} \mid \bar{B})>0$. Moreover, it is desirable that $P(\bar{A} \mid \bar{B})>P(\bar{C} \mid \bar{B})$.

It is worth nothing that in the aforementioned assumptions the system reasonably discards solutions that are actually consistent, but never accepts and validates solutions that are non-consistent, which is definitely a more critical aspect of validation in partially supervised problems. The best value for $C A$ obtained by the inductive SVM for $\mathbf{R}_{1}$ is denoted as $C A_{b e s t}$. We reasonably expect that the final value of $C A$ should be lower than $C A_{\text {best }}$ in the most cases, therefore, we suggest to fix the threshold $C A_{t h}=C A_{b e s t}-\varepsilon, \varepsilon \in[0.1 \div 0.2]$. The higher the threshold is, the more reliable the final results are but, at the same time, the number of the correct consistent solutions for the partially supervised problem discarded increases.

\section{Experimental Results}

In order to assess the effectiveness of the proposed approach, without loss of generality, we focused our attention on the analysis of remote-sensing images acquired over the same geographical area at different times. We carried out several experiments on a 
data set made up of two multispectral images acquired by the Thematic Mapper (TM) sensor of the Landsat 5 Satellite. The investigated area refers to Lake Mulargia (Italy). The two images were acquired in September $1995\left(\mathbf{R}_{1}\right)$ and in July $1996\left(\mathbf{R}_{2}\right)$, respectively. The available prior knowledge was used to derive a training and a test set for both the images, but in all the experiments, we assumed that only the information for the first date was available. We identified five land-cover classes that characterize the considered classification problem (see Table 1). We used the training set of $\mathbf{R}_{1}$ as $\mathbf{X}_{1} \quad(N=2249)$ and the test set of $\mathbf{R}_{2}$ as $\mathbf{X}_{2} \quad(M=1949)$. Five texture features in addition to the six TM channels (the thermal IR band was discarded) were taken into account, in order to exploit the non-parametric properties of the SVMs. The wellknown SMO algorithm [5] was employed in the learning phase of both the inductive SVMs and the proposed $\mathrm{T}^{2}$-SVMs (with proper modifications). For all the experiments we used Gaussian kernel functions.

An inductive SVM called SVM-1 was firstly trained over $\mathbf{R}_{1}$ : on the related test samples we obtained an Overall Accuracy and a Kappa coefficient equal to $95.33 \%$ and 0.938 , respectively. Table 2 reports the results obtained at $t_{2}$ over $\mathbf{X}_{2}$ by: i) SVM-1; ii) an inductive SVM trained using the available prior knowledge at $t_{2}$ (SVM-2) keeping the same parameters determined for SVM-1; iii) the $\mathrm{T}^{2}-\mathrm{SVM}$ (whose parameter values were $G=250, C^{*}=0.25, E=N \cdot 0.005$ and $P=N \cdot 0.035$ ), which exhibited the highest classification accuracy. The $\mathrm{T}^{2}-\mathrm{SVM}$ proved the effectiveness of the proposed technique exhibiting both an Overall Accuracy and a Kappa coefficient higher than those obtained by the inductive SVMs trained over $\mathbf{X}_{1}$. Furthermore, the accuracies are even slightly higher than those obtained with the inductive SVM trained over $\mathbf{X}_{2}$. This result is very important because it proves that $\mathrm{T}^{2}$-SVMs can model the addressed problem better than an inductive SVM trained on

Table 1. Number of Patterns in the Training and Test Sets for Both the September 1995 and July 1996 Images

\begin{tabular}{ccc}
\hline \multirow{2}{*}{ Class } & \multicolumn{2}{c}{ Number of patterns } \\
\cline { 2 - 3 } & Training & Test \\
\hline Pasture & 554 & 589 \\
Forest & 304 & 274 \\
Urban Area & 408 & 418 \\
Water Body & 804 & 551 \\
Vineyard & 179 & 117 \\
\hline
\end{tabular}

Table 2. Overall Accuracy and Kappa Coefficient over the Test Set of the July 1996 Image

\begin{tabular}{ccc}
\hline Classifier & Overall Accuracy & Kappa Coefficient \\
\hline SVM-1 & $87.84 \%$ & 0.843 \\
SVM-2 & $94.56 \%$ & 0.928 \\
$\mathrm{~T}^{2}$-SVM & $94.77 \%$ & 0.932 \\
\hline
\end{tabular}


the proper labeled patterns. It is worth nothing that the experiment with SVM-2 has been carried out only to assess the effectiveness and the reliability of the proposed technique: in partially supervised problems, in fact, a training set at $t_{2}$ is not available for definition. The classification accuracies of the $\mathrm{T}^{2}-\mathrm{SVM}$ approach resulted high and stable even into a large neighborhood of the optimal values for the transductive parameters (i.e., $G, C^{*}, P$ and $E$ ), thus proving the robustness of the proposed algorithm.

We also carried out several experiments for validating the proposed accuracy assessment procedure and the results obtained over 300 trials proved that it is particularly promising. The Kappa coefficient was chosen as the reference classification accuracy parameter $C A$, fixing $C A_{t h}=0.79$ (i.e. $\varepsilon=0.15$ ). The system moved from state $\bar{A}$ to state $\bar{B}$ in 260 cases. In the remaining 40 cases it moved to state $\bar{D}$. It is worth nothing that for simulating malfunctions of the $\mathrm{T}^{2}$-SVM learning process, we selected values for the transductive parameters very far from the optimal ones. The system did never obtain consistent solutions for $\mathbf{R}_{1}$ when it started from non-acceptable solutions for $\mathbf{R}_{2}$, thus satisfying the first requirement addressed in Section 4 . We point out that starting from state $\bar{B}$ the system went back to state $\bar{A}$ in the $54 \%$ of the cases. It is a very important result considering that 140 correct solutions for $\mathbf{R}_{2}$ were correctly identified without any prior training information, thus confirming the validity of the proposed accuracy assessment technique.

\section{Conclusion}

In this paper, a $\mathrm{T}^{2}-\mathrm{SVM}$ approach to partially supervised classification and the corresponding validation strategy have been presented. The results obtained on the investigated data set confirmed the effectiveness of both: i) the bi-transductive approach to partially supervised classification problems; ii) the accuracy assessment strategy for identifying correct solutions without having any training information.

\section{References}

1. L. Bruzzone and D. Fernàndez Prieto, "Unsupervised retraining of a maximum-likelihood classifier for the analysis of multitemporal remote-sensing images," IEEE Trans. Geosci. Remote Sensing, vol. 39, 2001.

2. L. Bruzzone and R. Cossu, "A multiple-cascade-classifier system for a robust and partially unsupervised updating of land-cover maps," IEEE Trans. Geosci. Rem. Sens, vol. 40, 2002.

3. T. Joachims, "Transductive inference for text classification using support vector machines," Proceedings of the 16th International Conference on Machine Learning, Bled, 1999.

4. Y. Chen, G. Wang and S. Dong, "Learning with progressive Transductive support vector machine," Pattern Recognition Letters, vol. 24, 2003.

5. N. Cristianini and J. Shawe-Taylor, An Introduction to Support Vector Machines, Cambridge University Press, 2000. 